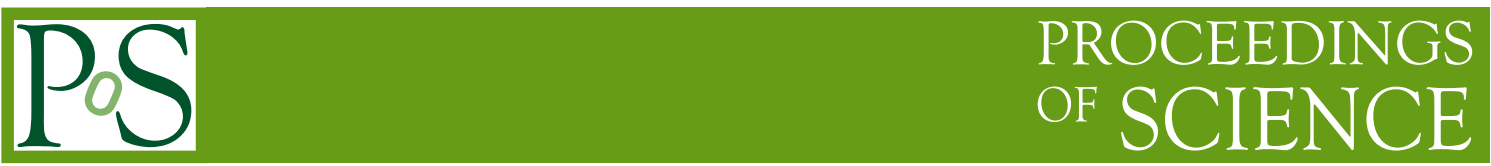

\title{
The CEVALE2VE case
}

\section{Arturo Sánchez*}

on behalf of the ATLAS collaboration

Universita degli Studi di Napoli Federico II and INFN

E-mail: arturosecern.ch

A virtual research and learning community can be a powerful tool for educational purposes. It has a wide range of possibilities for multi-institution participation, such as synchronous and asynchronous online engagement, decentralized student discussions and academic networking - as well as being cost effective. In this context, the CEVALE2VE virtual community (Centro de Altos Estudios de Altas Energías) is a Venezuelan initiative to support the new generation of researchers in High Energy Physics (HEP). Its goal is to contribute to the scientific dissemination of fundamental physics and the regional modernization of university education. The members of CEVALE2VE are a group of Venezuelan researchers, currently involved in projects related to the HEP field, and geographically located in different academic institutions of Europe and North America. The project involves several academic institutions in Venezuela and Colombia in order to reach a wide audience, and exploits current communications technologies, where data, software tools and information resources are shared. Several activities have been hosted by CEVALE2VE, including a series of public lectures, virtual visits to the ATLAS experiment, the implementation of a virtual course "Introduction to Particle Physics" for undergraduate students with the recent addition of dual Spanish-English material, and the supervision of masters theses in physics and internships in other fields like language studies. The use of the current technologies to share material and interact with the students creates a vibrant and participatory learning environment, having as its meeting point the www.cevale2ve.org portal.

38th International Conference on High Energy Physics

3-10 August 2016

Chicago, USA

* Speaker. 


\section{Origins and Vision}

The Centro de Altos Estudios de Altas Energías (CEVALE2VE)[1] is a virtual research and learning community created to steer the new generation of Venezuelan researchers in High Energy Physics (HEP)[2]. It also attempts to contribute to the scientific dissemination of fundamental physics and the regional modernisation of university education with the promotion of scientific communities in Latin America. The project organisation involves several academic institutions in Venezuela and Colombia in order to reach a wide regional audience. Although geographically scattered in different academic institutions of Europe and North America, CEVALE2VE integrates a group of Venezuelan researchers currently involved in projects related to the Large Hadron Collider (LHC)[3] of the European Organisation for Nuclear Research (CERN)[4]. Its main goal is to stimulate and widen Venezuelan physics postgraduate education and research, the first step of which has been the implementation of the virtual community course "Introduction to Particle Physics"[5].

Our main objective is to develop a virtual research and learning community with the aim of promoting the scientific dissemination, education and research in the field of HEP in the Venezuelan and Latin American scientific community:

Education and interest in research: positively influence and stimulate physics students interest in HEP research.

Awareness of opportunities: educate and invigorate the students with further study opportunities in physics and possible career paths in research.

Create networks and collaborations: in both directions! (Figure 1).

Modernization of the education and training: through the use of a web platform with elearning tools (Figure 2).

Policy making: working towards formalizing the involvement of Venezuelan institutions in HEP experiments.

\section{CEVALE2VE activities}

\subsection{Online Course}

We developed a course[5] for university students in physics (Figure 3). The ideal audience is masters and $\mathrm{PhD}$ students from the Latin American countries. The classes are in Spanish and the written materials are in English. The classes were possible using Google Hangouts[6] and transmitted and recorded in YouTube[7] (Figure 4). The program -with a duration of 60 hours- contains subjects from the Standard Model (SM), to accelerator and detector technology, data analysis and statistics.

The program is divided into Modules:

- Introduction to SM and physics Beyond the SM.

- Accelerator principles and particle detector principles.

- Introduction to statistics, data analysis and computer tools based in ROOT[8]. 


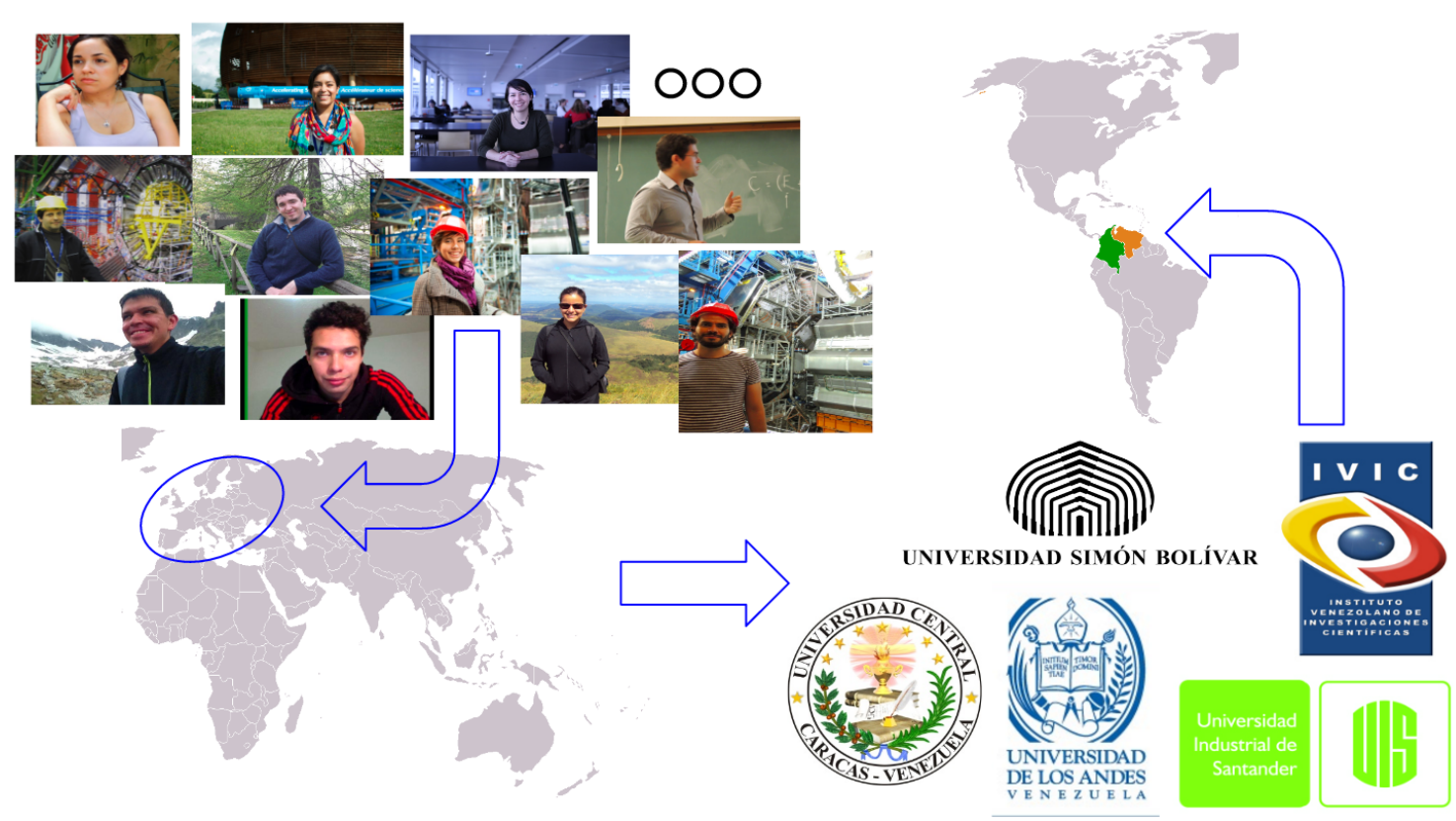

Figure 1: The CEVALE2VE members (left) are based mostly in European research centres while the students and their institutions are in Venezuela and Colombia (right). This is why an online platform has been the best way to maintain the collaboration between both regions.

- A series of seminars by invited speakers working in relevant areas of physics.

The opening edition of this course was carried out during September 2014 - February 2015[9], and the latest edition was during 2016 (from March to July). Moreover, this course was officially included in the postgraduate course portfolios of three public universities and research institutions, namely the Universidad Central de Venezuela (UCV), the Universidad Simón Bolívar (USB) and the Universidad de Los Andes (ULA), and informally followed by several students from the Universidad de Carabobo (UC), the Instituto Venezolano de Investigaciones Científicas (IVIC), the Universidad del Zulia (LUZ) and the Universidad Industrial de Santander (UIS).

\subsubsection{Student Supervisions}

In Physics: We have a Master student in physics developing a thesis in the evaluation of Dark Matter production in association with quarks or electroweak bosons in colliders like the LHC. She was part of our first session of the course in 2014-2015 and is currently enrolled in the UCV in Venezuela. This project is itself a test-field for newer technologies and tools in e-learning and data analysis. The plan is to finalize in the first quarter of 2017.

In Languages: This has been another beautiful example of the interdisciplinarity that can be involved. A university student in Languages was part of our group helping in the translation of all the classes of the first course into Spanish. The idea of this project is to have a dual Spanish-English set of classes and slides that allow us to expand the impact of the classes to other students. She got her bachelor's degree in July 2016 at ULA in Venezuela. 


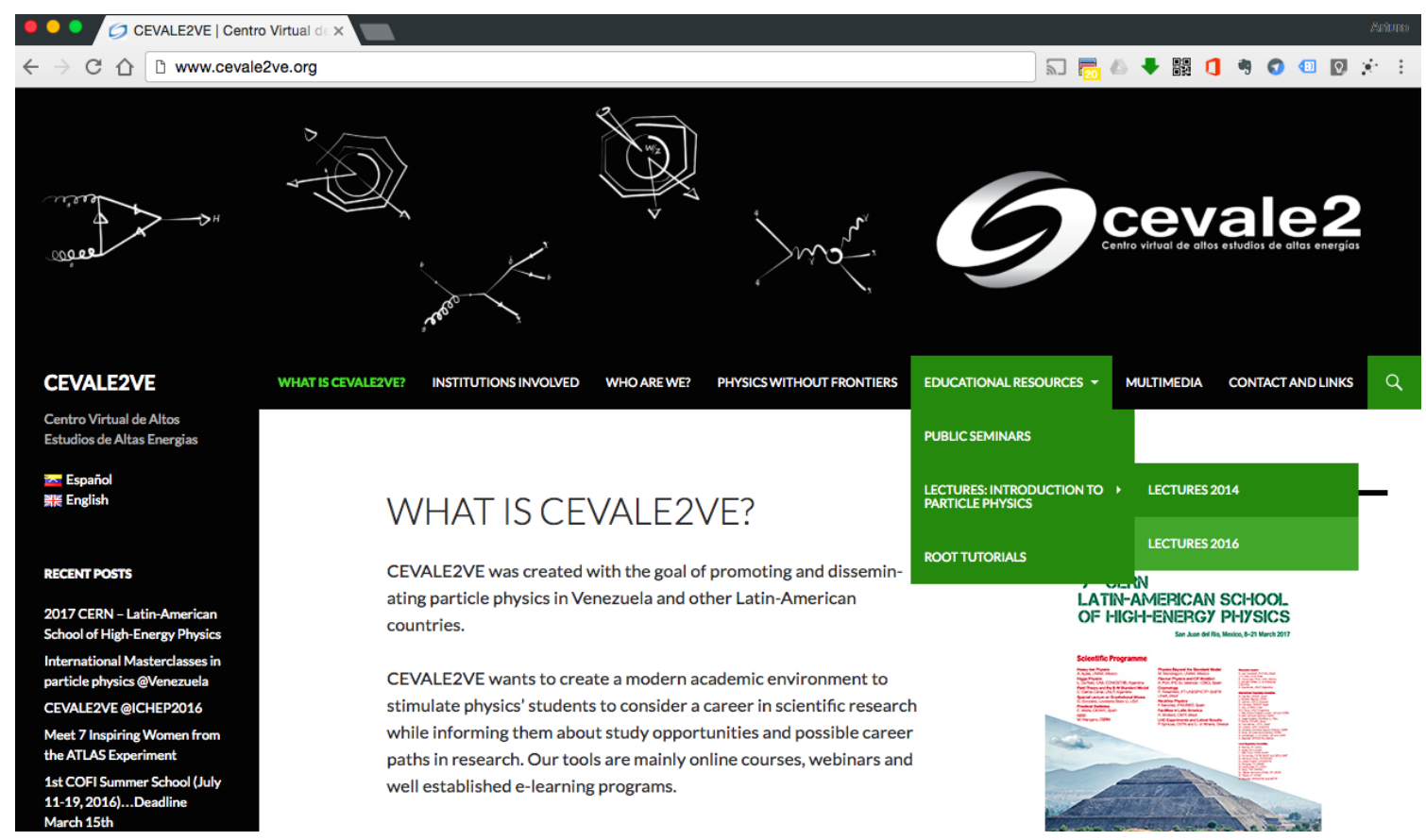

Figure 2: The CEVALE2VE web platform[1].

\subsection{Outreach}

The outreach events of CEVALE2VE are meant to be a way to disseminate the activities and the benefits of the scientific activities taking place in international organizations like CERN and others around the world. At the same time to let the nationals of Latin American countries know how they contribute into this global scientific enterprise. Some are public lectures or seminars in Spanish presented by CEVALE2VE members or invited physicists of other areas to bring a broader overview of the state of the art in fundamental physics research. Like the classes of the particle physics course, the seminars and presentations are recorded (when possible) and available on a dedicated channel (Figure 4).

More recently, for the first time we took the International Particle Physics Outreach Group (IPPOG)[10] Masterclasses[11] and ATLAS[12] Virtual Visit[13] to Venezuela. This had a very good impact with the university community there and thanks to our collaborators in Colombia we developed an outreach event of two weeks in four cities in Colombia and Venezuela[14]. This was possible thanks to the participation of the ATLAS Collaboration[15] and the International Centre for Theoretical Physics (ICTP)[16] Physics Without Frontiers program[17].

Finally, we try to do some diplomacy. We have been looking to communicate the projects and results to diplomatic members of the Venezuelan embassies around the world in order to get a more formal agreement between the country's authorities and CERN. We understand this is a slow process and we keep doing it constantly. 


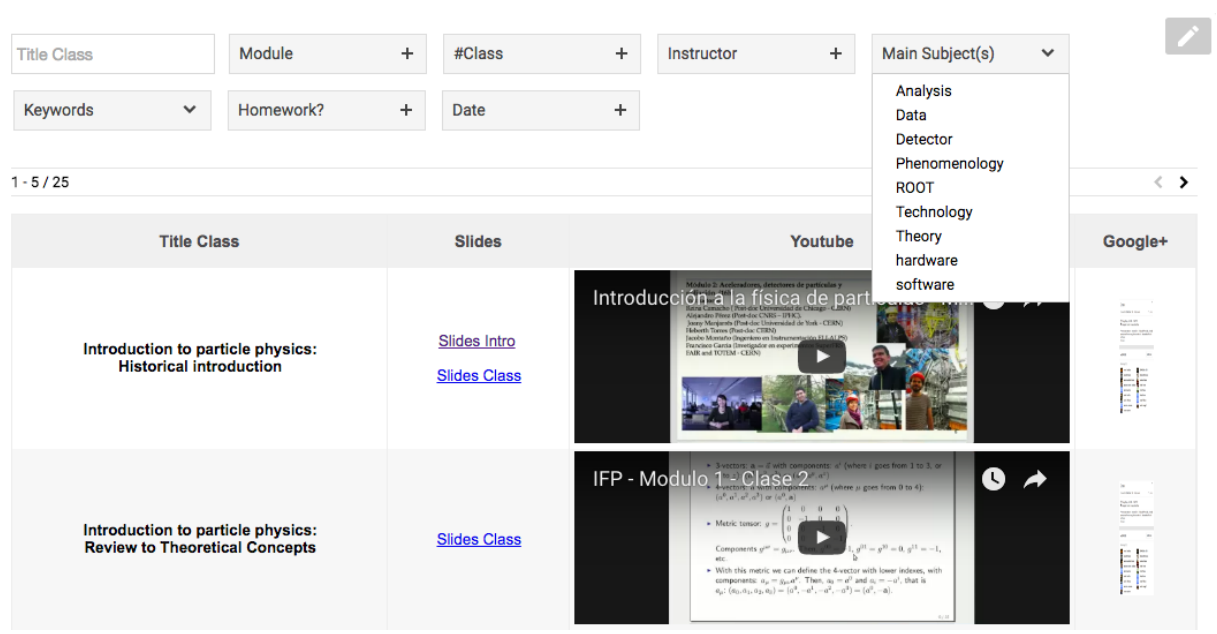

Figure 3: Web-based repository of all of the online particle physics courses and seminars. The repository is hosted into the CEVALE2VE web platform and it contains the classes material and metadata, links to the online events and videos.

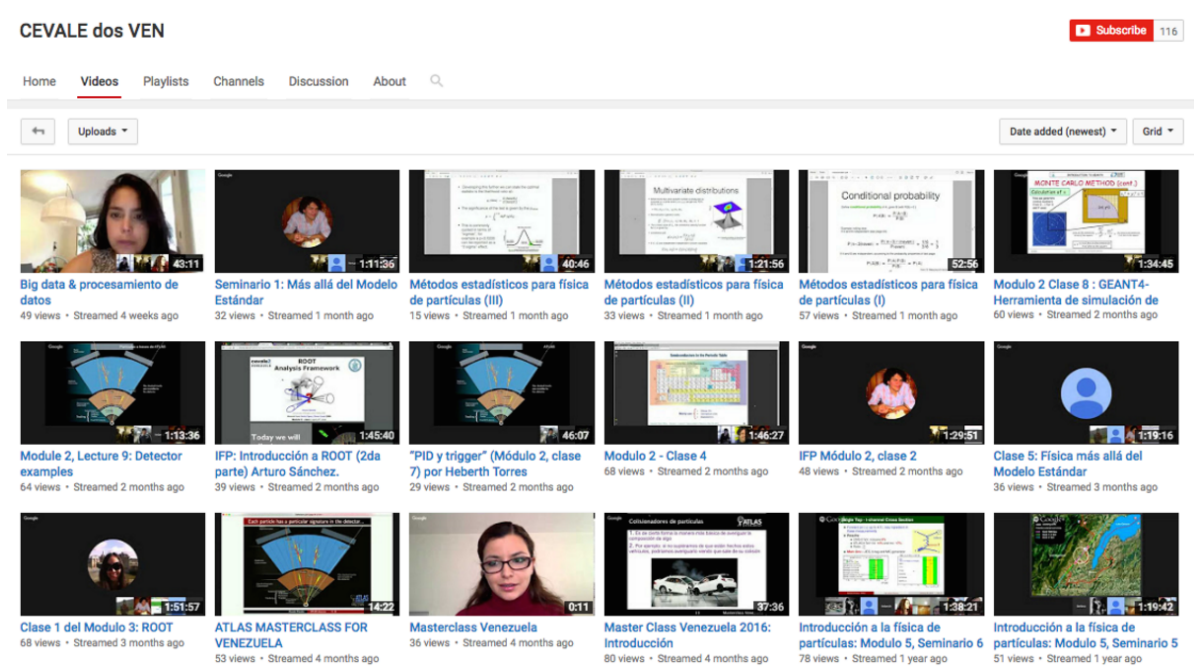

Figure 4: The CEVALE2VE YouTube channel, which contains more than 70 hours of online audio-visual content and 9000 views and dozens of comments in the tutorials.

\section{Current Status and the Future}

The CEVALE2VE is a HEP educational and outreach project for Latin America. With the help of a lot of people on both sides of the ocean, we developed a course in particle physics as an "excuse" to create and strengthen ties between members of the scientific community. Several students have got a good start in their advanced studies thanks to this collaboration and their incredible energies: some of our students have participated in the HEP CERN-LA school and CERN/DESY summer student programs. In 2017 we will again be offering our "Introduction to Particle Physics" course. We seek to extend our university community and our partnerships with other institutions and individual collaborators in order to reach more students, more regions and more members that can make of CEVALE2VE a truly international collaboration in its own right. 


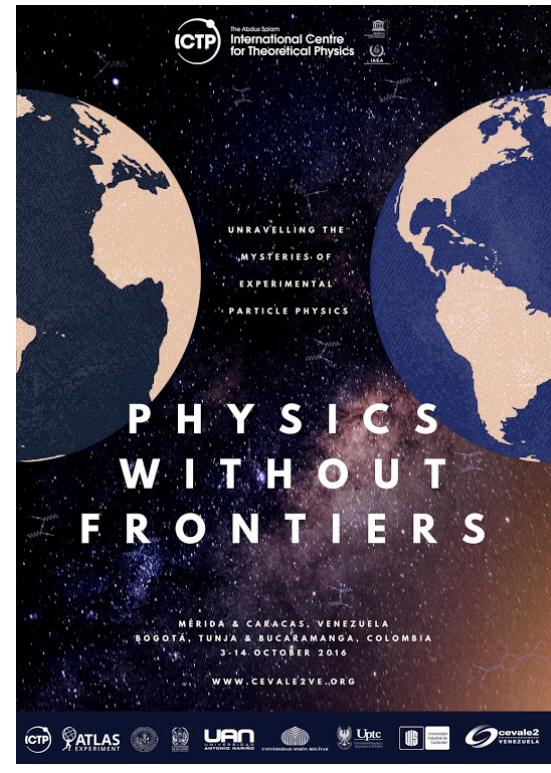

Figure 5: The CEVALE2VE + ICTP Physics Without Frontiers + ATLAS Figure 6: Some of the students visited by the CEVALE2VE memoutreach project, October 2016.
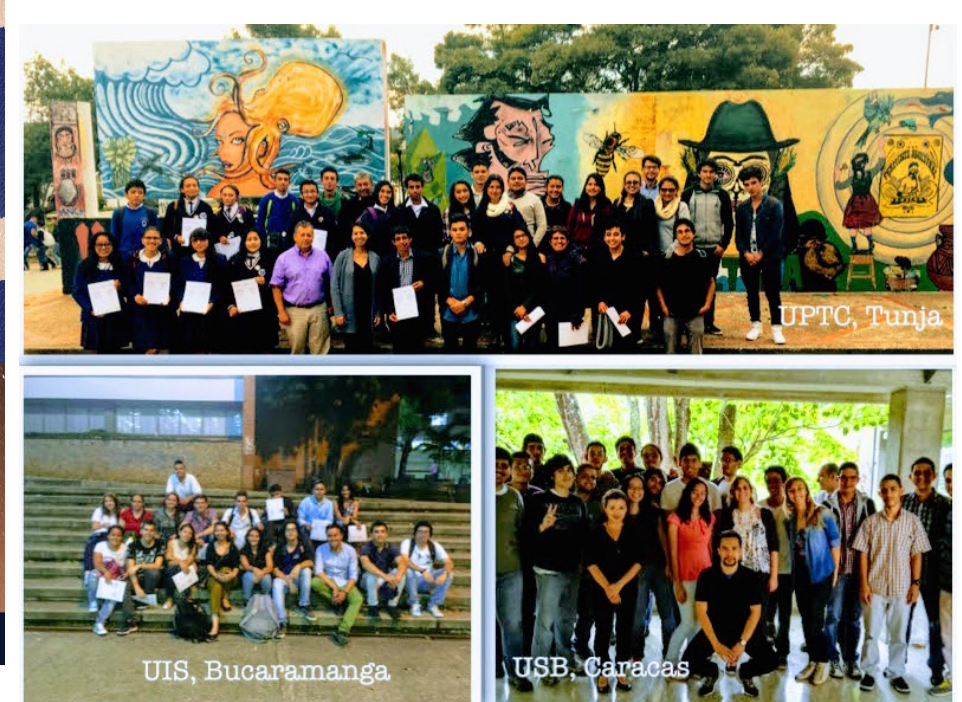

bers during the two week Colombo-Venezuelan event.

\section{References}

[1] CEVALE2VE Website - www.cevale2ve.org

[2] High Energy Physics - Wikipedia https://en.wikipedia.org/wiki/high-energy-physics

[3] The Large Hadron Collider (LHC) - http://home.cern/topics/large-hadron-collider

[4] European Organization for the Nuclear Research (CERN) - http://home.cern

[5] CEVALE2VE online course http://www.cevale2ve.org/course

[6] Google Hangouts - https://hangouts.google.com

[7] CEVALE2VE YouTube video hosting service - www.youtube.com/cevale2ve

[8] Brun R and Rademakers F. ROOT - An Object Oriented Data Analysis Framework Nucl. Inst. \& Meth. in Phys. Res. A 389 pp 81-86, 1996.

[9] EPS 2015 presentation and document - ATL-OREACH-PROC-2016-002.

[10] International Particle Physics Outreach Group (IPPOG) - http://ippog.web.cern.ch

[11] IPPOG Masterclasses program - http://physicsmasterclasses.org

[12] G. Aad et al. The ATLAS Experiment at CERN LHC, ATLAS Collaboration, JINST 3 (2008) 03.

[13] ATLAS Virtual Visits - http://atlas-live-virtual-visit.web.cern.ch/atlas-live-virtual-visit

[14] Photographic records of project CEVALE2VE + PWF + ATLAS, October 2016 https://cds.cern.ch/record/2235879

[15] The ATLAS Open Data portal - http://opendata.atlas.cern

[16] International Centre for Theoretical Physics (ICTP) - https://www.ictp.it

[17] ICTP Physics Without Frontiers program - https://www.ictp.it/physics-without-frontiers 